\title{
Effects of transcranial direct current stimulation (tDCS) on posture, movement planning, and execution during standing voluntary reach following stroke
}

\author{
Chieh-ling Yang ${ }^{1,3,4^{*}} \mathbb{C}$, Alon Gad ${ }^{1}$, Robert A. Creath ${ }^{1,5}$, Laurence Magder ${ }^{2}$, Mark W. Rogers ${ }^{1}$
} and Sandy McCombe Waller ${ }^{1,6}$

\begin{abstract}
Background: Impaired movement preparation of both anticipatory postural adjustments and goal directed movement as shown by a marked reduction in the incidence of StartReact responses during a standing reaching task was reported in individuals with stroke. We tested how transcranial direct current stimulation (tDCS) applied over the region of premotor areas (PMAs) and primary motor area (M1) affect movement planning and preparation of a standing reaching task in individuals with stroke.

Methods: Each subject performed two sessions of tDCS over the lesioned hemisphere on two different days: cathodal tDCS over PMAs and anodal tDCS over M1. Movement planning and preparation of anticipatory postural adjustment-reach sequence was examined by startReact responses elicited by a loud acoustic stimulus of $123 \mathrm{~dB}$. Kinetic, kinematic, and electromyography data were recorded to characterize anticipatory postural adjustment-reach movement response.

Results: Anodal tDCS over M1 led to significant increase of startReact responses incidence at loud acoustic stimulus time point $-500 \mathrm{~ms}$. Increased trunk involvement during movement execution was found after anodal M1 stimulation compared to PMAs stimulation.

Conclusions: The findings provide novel evidence that impairments in movement planning and preparation as measured by startReact responses for a standing reaching task can be mitigated in individuals with stroke by the application of anodal tDCS over lesioned M1 but not cathodal tDCS over PMAs. This is the first study to show that stroke-related deficits in movement planning and preparation can be improved by application of anodal tDCS over lesioned M1.

Trial registration ClinicalTrial.gov, NCT04308629, Registered 16 March 2020—Retrospectively registered, https://www. clinicaltrials.gov/ct2/show/NCT04308629
\end{abstract}

Keywords: Transcranial direct current stimulation, startReact, Motor preparation, Postural control, Reach, Stroke

\footnotetext{
*Correspondence: chieh-ling.yang@ubc.ca

1 Department of Physical Therapy and Rehabilitation Science, University of Maryland School of Medicine, 100 Penn Street, Baltimore, MD 21201, USA

Full list of author information is available at the end of the article
}

\section{Introduction}

StartReact (SR) responses triggered by a loud acoustic stimulus (LAS) during the planning and preparation of goal intended actions have been used to probe the state of brainstem neuronal excitability related to posture and 
movement sequencing $[1,2]$. Abnormal posture and movement planning and preparation as shown by an absence of SR responses during standing reaching have been found in previous studies [3, 4]. Premotor areas (PMAs) such as supplementary motor areas and premotor cortex are thought to be involved in posture and movement planning $[5,6]$. In preparation for a movement, the neural pathways originating from PMAs to the spinal cord via the reticular formation modulate spinal circuitry through inhibitory effects in order to prevent premature release of the movement [7]. Previous studies also suggested that damage to the premotor cortex following stroke $[6,8]$ or temporary inhibition by transcranial magnetic stimulation (TMS) over the supplementary motor areas of healthy subjects [7] impair the anticipatory postural adjustments (APAs) preparation during voluntary stepping. Furthermore, animal studies $[9,10]$ have shown the activation in neurons in subcortical pontomedullary reticular formation (PMRF) were related to the APAs prior to the reaching movement. The signals for the APAs were possibly generated from higher cortical level such as PMAs via cortico-reticular pathway to the PMRF. We proposed that PMAs normally have a modulatory role in SR responses through inhibitory input to brainstem motor circuits and/or spinal cord via the PMRF. Hence, abnormal hyperexcitability in PMAs due to chronic stroke [11] may lead to excessive inhibition of the PMRF and/or spinal cord resulting in an absence of and/or reduced magnitude of SR responses and a disruption of the normal sequencing between posture and movement [4].

Cortical excitability can be modulated by the application of weak continuous direct electrical current over a specific location of the head by noninvasive transcranial direct current stimulation (tDCS) [8]. Depending on the direct current polarity, $\mathrm{tDCS}$ can either up-regulate neuronal excitability using anodal tDCS or down-regulate it using cathodal $\mathrm{tDCS}$ by hyperpolarizing or depolarizing the membrane potentials $[8,12]$. Many studies have demonstrated beneficial effects of applying tDCS over M1 on arm, hand, and lower limb motor performance in individuals with stroke [13-18]. However, only one recent study demonstrated that SR response in ankle dorsiflexion, wrist flexion, and automatic postural responses could be facilitated by applying anodal tDCS over M1 in healthy subjects [19]. No studies have used tDCS over PMAs as a target for neuromodulation therapy to augment posture and movement planning, preparation, and execution following stroke.

The purpose of this study was to determine the modulatory role of the PMAs on SR responses following cathodal tDCS over PMAs in persons with stroke. Knowing that PMAs have projections to the M1, we included anodal tDCS over M1 as a control condition to validate that PMAs stimulation has additional modulatory effects. Our hypothesis was that applying cathodal tDCS over PMAs will reduce the neuronal excitability in PMAs thereby helping to improve posture and movement planning and preparation in persons with stroke, as measured by increased incidence and faster onset of the SR responses.

\section{Material and methods \\ Subject}

Participants were recruited through convenience sampling. The recruitment process flowchart is displayed in Fig. 1. Data were collected from 10 individuals with stroke (Table 1). Inclusion criteria were unilateral cortical or white matter subcortical stroke, age 40 years and older, $\geq 6$ months post ischemic stroke or $\geq 12$ months post hemorrhagic stroke, residual arm hemiparesis as indicated by Fugl-Meyer Upper Extremity [20] score between 20 and 65, and having the ability to perform reaching movements with the paretic arm in standing without an assistive device. Exclusion criteria included stroke involving bilateral hemisphere, brainstem or cerebellum, any medical condition precluding participation in testing, and other health conditions affecting balance and upper extremity movement function beyond the effects of stroke. Participants were also excluded if they did not meet the TMS safety criterion including having implantable medical devices, history of seizures, taking medications to reduce anxiety, sedatives, and seizure, and pregnancy. All participants gave written informed consent to participate, and the study was approved by the Institutional Review Board at the University of Maryland Baltimore (HP-00064894). Participants were recruited from October 2016 and the data collection of all participants was completed by Oct 2017. There was no deviation from the study protocol. The study was retrospectively registered on ClinicalTrial.gov due to the lack of knowledge about trial registration before enrolment of participants. We confirmed that all ongoing and related trials for this intervention are registered.

\section{Transcranial magnetic stimulation}

Motor hotspots were located by using single-pulse TMS delivered by a Magstim 200 stimulator (Magstim Company, Dyfed, UK) using a figure-of-eight coil (70-mm) for biceps brachii and a double cone coil (110-mm) for tibialis anterior. For subjects who had absent motor evoked potentials (MEP) of the affected side, the mirrored location of the nonaffected side hotspot was used to determine the hotspot for the affected side. The active motor threshold was determined while the subjects exerted a force of $20 \%$ maximum voluntary isometric contraction 


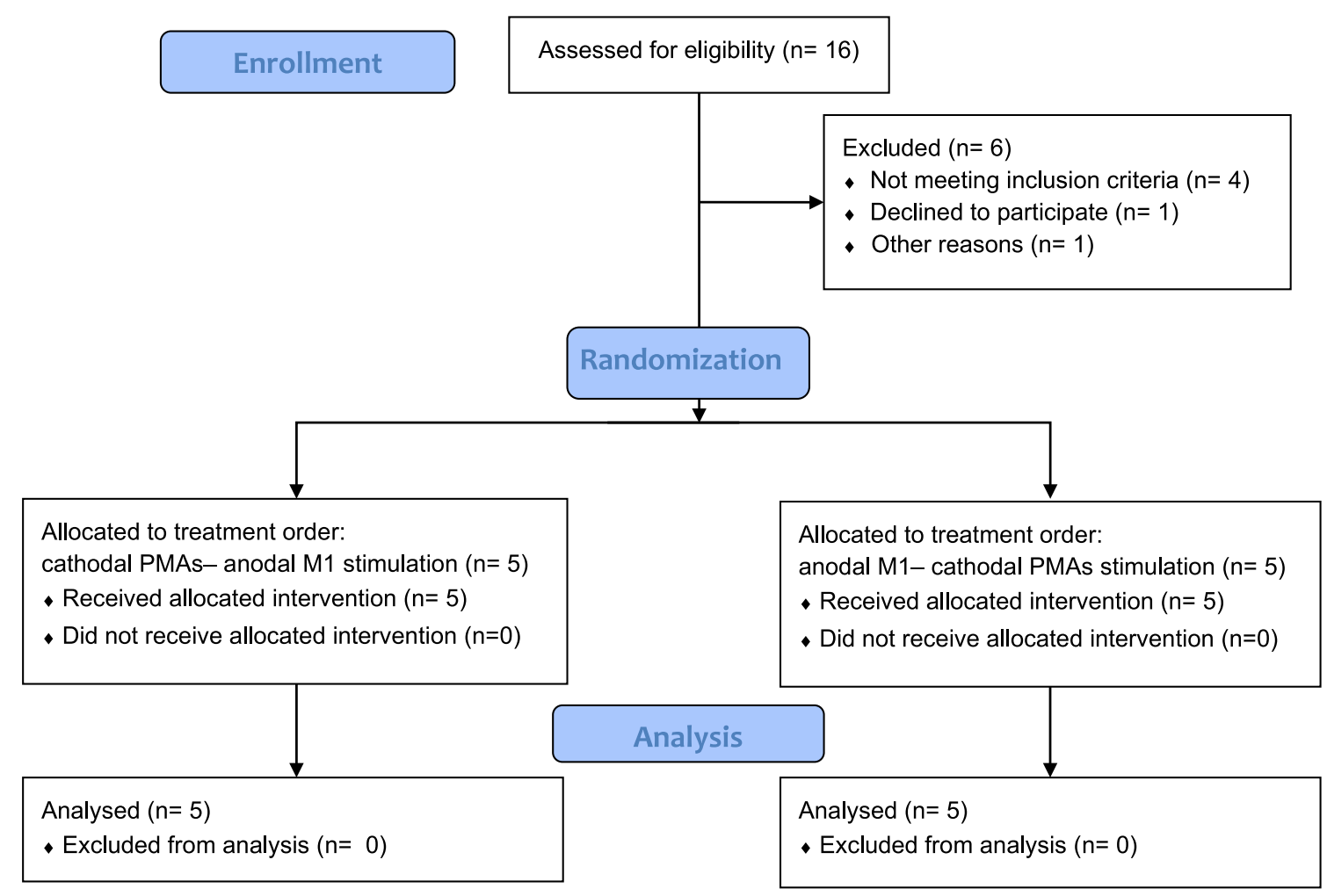

Fig. 1 The CONSORT flow diagram showed the recruitment process

Table 1 Demographic characteristic

\begin{tabular}{|c|c|c|c|c|c|c|c|}
\hline Subject id & Age, years & Sex & Time Poststroke, y & Lesion location & Side of paresis & Dominant side & FM-UE (/66) \\
\hline$\# 1$ & 75.73 & M & 14.00 & Cortical and subcortical & $\mathrm{R}$ & $R$ & 49 \\
\hline \#2 & 63.36 & M & 5.91 & Cortical and subcortical & $\mathrm{R}$ & $\mathrm{R}$ & 39 \\
\hline \#3 & 77.63 & M & 20.51 & Cortical & L & L & 33 \\
\hline \#4 & 62.58 & $\mathrm{~F}$ & 7.55 & Cortical and subcortical & L & $\mathrm{R}$ & 30 \\
\hline \#5 & 68.14 & M & 8.81 & Cortical and subcortical & L & $\mathrm{R}$ & 62 \\
\hline \#6 & 70.33 & $\mathrm{~F}$ & 16.40 & Subcortical & $\mathrm{R}$ & $\mathrm{R}$ & 26 \\
\hline \#7 & 74.23 & $\mathrm{~F}$ & 51.26 & Subcortical & L & L & 36 \\
\hline \#8 & 64.10 & M & 0.97 & Subcortical & $\mathrm{R}$ & $\mathrm{R}$ & 65 \\
\hline \#9 & 55.99 & M & 2.26 & Subcortical & $\mathrm{R}$ & $\mathrm{R}$ & 65 \\
\hline$\# 10$ & 79.29 & M & 1.29 & Subcortical & L & $\mathrm{R}$ & 55 \\
\hline Mean (SD) & $69.13(7.61)$ & $7 \mathrm{M} / 3 \mathrm{~F}$ & $12.00(15.00)$ & $\begin{array}{l}1 \text { Cortical/5 Subcortical/4 } \\
\text { Cortical and subcortical }\end{array}$ & $5 \mathrm{R} / 5 \mathrm{~L}$ & $8 \mathrm{R} / 2 \mathrm{~L}$ & $46.00(15.06)$ \\
\hline
\end{tabular}

FM-UE Fugl-Meyer Upper Extremity Score

(MVIC) of each muscle [25]. The active motor threshold was defined as the lowest stimulus intensity that could evoke a MEP (about $200 \mu \mathrm{V}$ ) in 5 out of 10 consecutive trials during isometric contraction of the tested muscle. A hand-held dynamometer (Chatillon DFX-200 Digital Force Gauge, Itin Scale Co., Inc., Brooklyn, NY) was used to measure the MVIC. The MVIC is defined as the average of 3 measurements of each tested muscle. For each MEP measurement, the assessor holding the dynamometer visually verified if the force reached the level of $20 \%$ MVIC and instructed the subjects to hold the level of force exertion. Changes in cortical excitability as measured by MEPs were measured 10 times at the hotspots of the biceps brachii and tibialis anterior with 
an intensity of the $120 \%$ of active motor threshold at $20 \%$ of MVIC. A neuronavigation system (Brainsight Version 2, Rogue Research Inc., Montreal, Canada) was used to confirm that the same hotspots were used.

\section{Transcranial direct current stimulation}

TDCS was applied by an iontophoresor (Chattanooga Ionto, Salt Lake City, Utah). The stimulating electrode was placed at the midpoint of the supplementary motor area and premotor cortex for PMAs stimulation (Fig. 2a). Supplementary motor area was defined as $1.8 \mathrm{~cm}$ anterior to the measured location of $\mathrm{Cz}$ [21]. Premotor cortex was defined as $2.5 \mathrm{~cm}$ anterior to the motor hotspot of the biceps brachii [22]. For M1 stimulation, the stimulating electrode was placed at the midpoint of upper and lower extremity M1 where TMS elicits twitches in the biceps brachii and tibialis anterior of the limb respectively (Fig. 2b). The reference electrode was placed on the forehead above the contralateral orbit. Custom-made tDCS electrodes of $15 \mathrm{~cm}^{2}(3 \mathrm{~cm} \times 5 \mathrm{~cm})$, made of carbon-microfiber material, were thoroughly hydrated by saline $(0.9 \% \mathrm{NaCl})$ and secured over the subject's head. One-session of tDCS was administered at an amplitude of $1 \mathrm{~mA}$ for $20 \mathrm{~min}$ while the subjects were sitting on a chair.

\section{Instructed-delayed paradigm}

A visually cued delayed-response paradigm was used to examine the transition from a stationary standing posture to the rapid initiation of reaching (Fig. 3). Task instruction stimuli were presented using LED lights placed at eye level $3 \mathrm{~m}$ in front of the subject. A precue (center) light was presented followed by the imperative "go" cue light with an inter-stimulus delay of $2.5 \mathrm{~s}$. The target ball was placed at $65 \%$ of subject's height and $10 \mathrm{~cm}$ beyond subjects' maximal reach distance of the paretic arms.
Subjects were instructed to reach with their paretic arms "as quickly as possible" in response to the "go" cue. An LAS (123 dB, $1 \mathrm{kHz}, 40 \mathrm{~ms})$ delivered by a horn speaker (HS-17 T; MG Electronics) placed $30 \mathrm{~cm}$ behind subject's head.

In each testing, subjects performed 65 trials including three conditions: control reach trials, LAS reach trials, and control LAS trials. The order of presentation of LAS (i.e., LAS reach trials and control LAS trials) and control reach trials was partly randomized with the exception that the LAS was not presented during the first five trials and no more than two trials with LAS were presented in a row. Condition 1, control reach trials (45 trials): these trials consisted of standing reach movements performed with no LAS presented. Condition 2, LAS reach trials ( 3 time points $\times 5$ trials, 15 trials): these trials consisted of standing reach movement performed with the LAS presented at one of the three time points: $-500,-200$, or $0 \mathrm{~ms}$ relative to the "go" cue. These time-points were selected based on past normative studies showing progressive increases in the incidence and magnitude of SR responses during this time window reflecting motor preparation [26]. In addition, Condition 3, control LAS trials (5 trials): these trials were collected in which an LAS was delivered during inter-trial standing rest period without reach and without the presence of the precue and go cue, serving as catch trials to verify that in the absence of movement plan, an LAS did not elicit SR response. The number of trials with LAS were kept at $33 \%$ of all trials to avoid habituation $[27,28]$.

\section{Experimental design}

Each subject performed two sessions of tDCS over the lesioned hemisphere on two different days separated by at least a 48-h interval: cathodal tDCS over PMAs and anodal tDCS over M1. Knowing that PMAs have

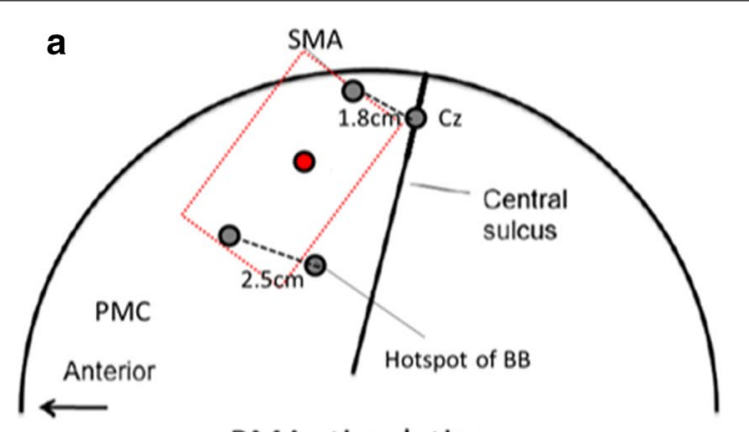

PMA stimulation

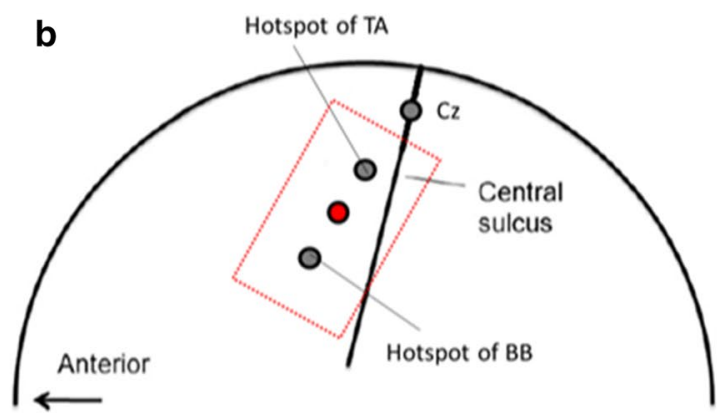

M1 stimulation

Fig. 2 Illustration of the stimulation electrode placements for a PMAs stimulation and $\mathbf{b}$ M1 stimulation during tDCS. SMA, supplementary motor area; PMC, premotor cortex. Supplementary motor area was defined as $1.8 \mathrm{~cm}$ anterior to the measured location of Cz [21]. Premotor cortex was defined as $2.5 \mathrm{~cm}$ anterior to the motor hot spot of the BB [22]. The hotspot of the BB is normally situated approximately $3 \mathrm{~cm}$ lateral and $2 \mathrm{~cm}$ in front of the Cz [23] and the hotspot of the TA is situated approximate $2 \mathrm{~cm}$ lateral and $1 \mathrm{~cm}$ in front of the Cz [24] 
a
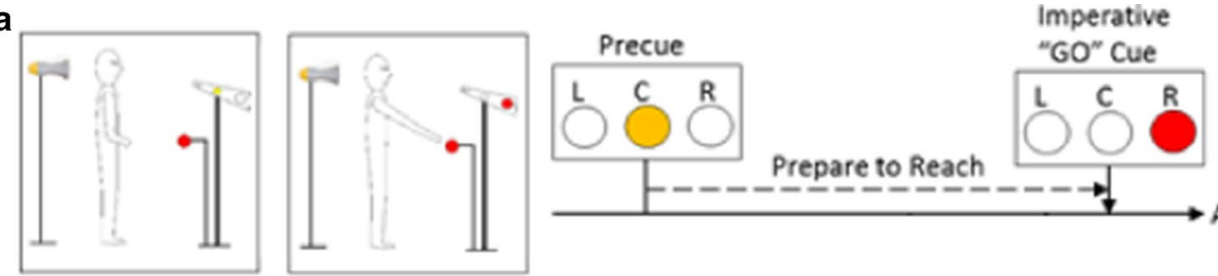

APA-Reach sequence

b
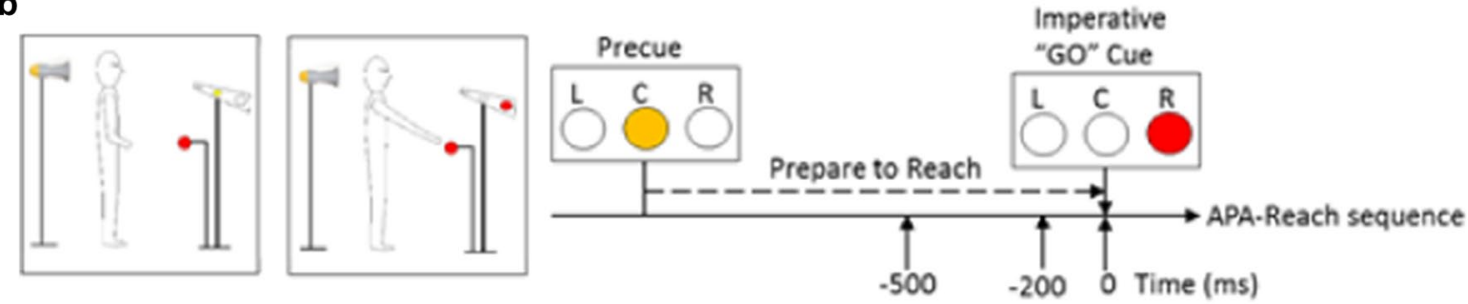

C

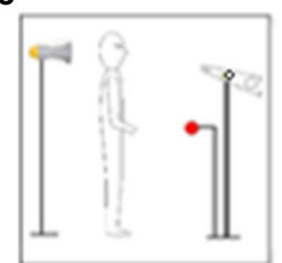

Inter-trial rest period

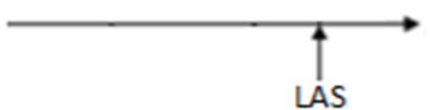

Fig. 3 Examples of $\mathbf{a}$ a right arm control reach trial, $\mathbf{b}$ a right arm LAS reach trial, and $\mathbf{c}$ a control LAS trial

projections to the M1, the M1 condition was used to validate that PMAs stimulation has additional modulatory effects. The order of PMAs and M1 stimulation was randomized. Each day consists of a pre-tDCS test, one tDCS session, and a post-tDCS test (Fig. 4). The pretDCS testing included finding the hotspots and MEP measurements followed by the standing reaching trials to examine the SR responses. The post-tDCS testing was a repeat of the pre-tDCS testing.

\section{Data acquisition}

Kinetic data including ground reaction forces and moment were collected from two force platforms (AMTI, Watertown, MA) placed beneath the right and left feet at a collection frequency of $600 \mathrm{~Hz}$. Kinematic data were collected at $120 \mathrm{~Hz}$, using a 10-camera Vicon motion analysis system (VICON, Los Angeles, CA). These data were filtered with a low pass, $4^{\text {th }}$ order Butterworth digital filter with a cutoff frequency at $10 \mathrm{~Hz}$ [29]. Reflective markers were placed bilateral on subject's body (see our previous study [4] for detailed placement). Kinematic computations of joint centers were performed using a model [30] written in commercially available software (BodyBuilder, Vicon, Centennial, CO). The muscle activity was recorded from anterior deltoid and biceps brachii of the reaching arm muscle and bilateral tibialis anterior, with a wireless EMG system TeleMyo ${ }^{\mathrm{TM}}$ Direct Transmission System (NORAXON, Scottsdale, AZ) using bipolar

\begin{tabular}{|c|c|c|c|c|}
\hline Pre-TMS & Pre-testing & tDCS session & Post-TMS & Post-testing \\
\hline & $\begin{array}{l}\text { StartReact posture and } \\
\text { movement responses }\end{array}$ & $1 \mathrm{~mA}, 20 \mathrm{mins}$ & $\begin{array}{l}\text { MEP (20\% MVIC, } \\
120 \% \text { AMT) }\end{array}$ & $\begin{array}{l}\text { StartReact posture and } \\
\text { movement responses }\end{array}$ \\
\hline $\begin{array}{l}\text { MEP ( } 20 \% \text { MVIC, } \\
120 \% \text { AMT) }\end{array}$ & T.7. & & & ए1. $\pi^{\circ}$ \\
\hline
\end{tabular}

Fig. 4 Flowchart of the experimental procedure for each visit. TMS, transcranial magnetic stimulation; tDCS, transcranial direction current stimulation; BB, biceps brachii; TA, tibialis anterior; MEP, motor-evoked potential; MVIC, maximum voluntary isometric contraction; AMT, active motor threshold 
$\mathrm{Ag}-\mathrm{AgCl}$ surface electrodes. All electrodes placements followed the recommendations of SENIAM (https:// www.seniam.org) [31]. Raw EMG signals were sampled at $1500 \mathrm{~Hz}$. The data for the standing reaching task was bandpass filtered between $30-500 \mathrm{~Hz}$ with a 5 th order Butterworth filter with Matlab program filtfilt, full-wave rectified, and low-pass filtered $(10 \mathrm{~Hz}$ Butterworth 4th order) for smoothing purposes. Custom-written Matlab programs (The MathWorks, Inc., Natick, MA) were used to process kinetic, kinematic, and EMG data and all data were verified by visual inspection.

\section{Data analysis}

\section{Incidence of StartReact response following LAS}

Movement planning and preparation were examined using the presence of SR responses (Fig. 5). The incidence of SR responses when the LAS was applied at $-500 \mathrm{~ms}$ and $-200 \mathrm{~ms}$ was reported. The SR responses for the trials when the LAS was presented at the "go" cue were not determined since the responses evoked by the LAS were possibly intermingled with the responses to the imperative "go" signal. To be considered a SR response in the APA or reach, the occurrence of components of APA or reach were required to be met within one of the following time windows: between the LAS and the go cue, or an early onset of $<3$ SDs from the average onset in the control reach condition. The components for an APA response are an initial posterior shift in the center of pressure and an early EMG burst in tibialis anterior before the onset of reach. The components for a reach response are an anterior movement of hand and an EMG burst in anterior deltoid.

\section{APA-reach performance}

APA and reach onset were defined as the onset of the posterior center of pressure displacement and the onset of the anterior wrist joint center movement with a threshold of $5 \%$ peak velocity, respectively. The onset times of muscle activation was calculated based on changes of $>3$ SDs for at least $100 \mathrm{~ms}$ from the mean signal recorded before the "go" cue or LAS and a continuous increase of muscle activity was seen. The onset times were verified by visual inspection [32].

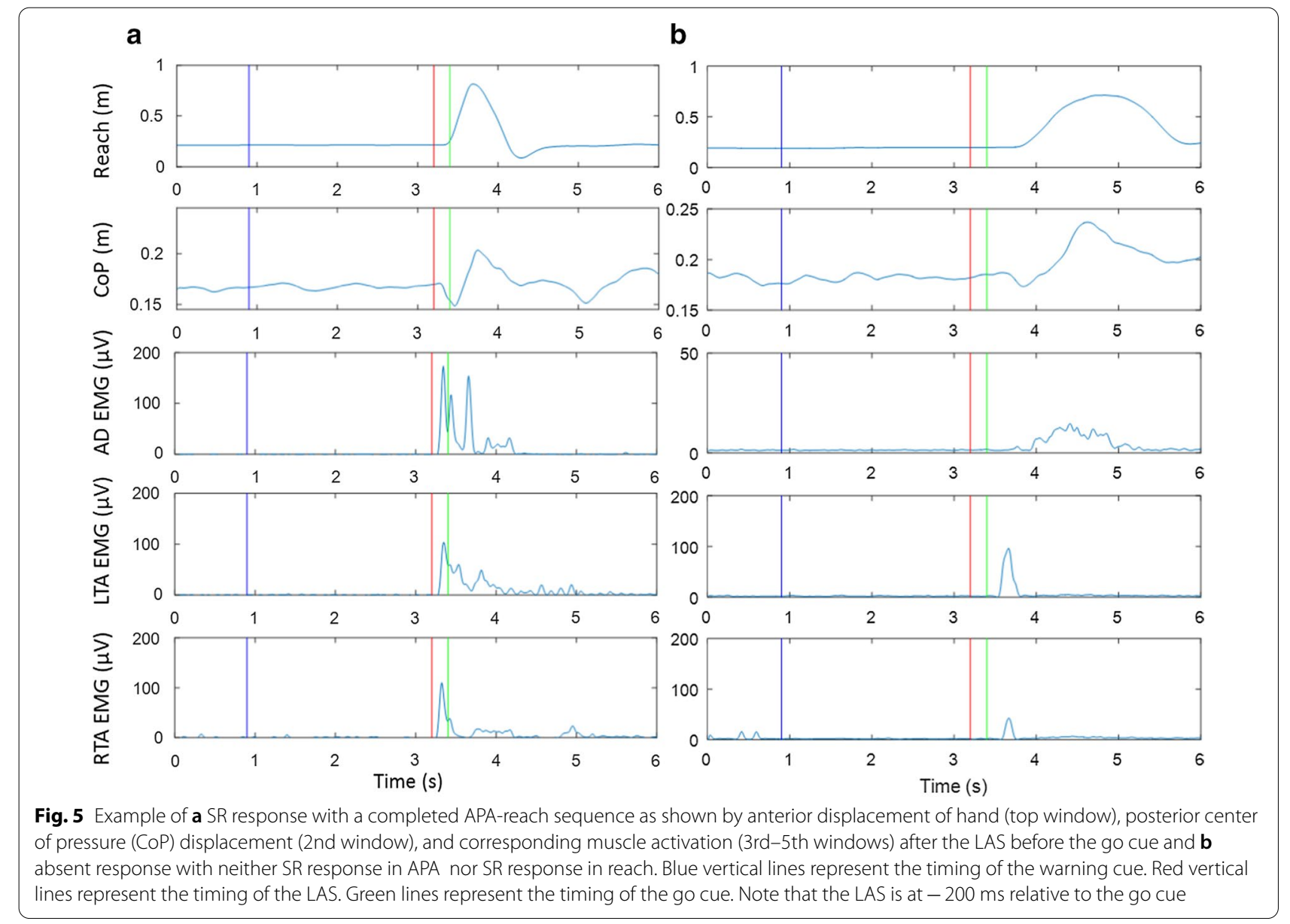




\section{Trunk contribution during movement execution}

Trunk flexion was determined by the angular displacement of the line joining the reaching shoulder and the hip joint center on the same side in the sagittal plane at maximum reach normalized by reach distance. Trunk rotation was determined from the angular displacement of the line connecting both shoulders in the horizontal plane in the direction of the reach at maximum reach normalized by reach distance. Pelvic rotation was determined from the angular displacement of the line connecting both hip joint centers in the horizontal plane in the direction of the reach at maximum reach normalized by reach distance. Trunk-pelvic rotation difference was determined from the difference between trunk and pelvic angular displacement at maximum reach normalized by reach distance.

\section{Neurophysiological measurement}

MEP amplitude was measured by the peak-to-peak EMG amplitude elicited by the TMS.

\section{Statistical analysis}

A linear mixed-effects model using Stimulation (cathodal PMAs vs. anodal M1) and LAS condition (LAS at $-500 \mathrm{~ms},-200 \mathrm{~ms}, 0 \mathrm{~ms}$ relative to the go, and control reach) as fixed factors, and subjects as a random factor was performed to test the effect of cathodal PMAs vs. anodal M1 stimulation adjusting for LAS timing on prepost change of outcome variables. The model included the main effects of Stimulation, LAS condition, Stimulation $\times$ LAS condition interaction, and a random intercept for subjects. Prior to analysis, proportion variables (e.g., incidence of SR response) were corrected for normality using an arcsine square root transformation. Bonferroni adjusted test was used for all post hoc comparison. All outcome variables except for MEP amplitude were transformed and presented as Post-Pre change values. Difference in pre vs. post MEP amplitude was examined by paired t-tests. All statistical analyses were performed by SPSS v.22 (IBM, Armonk, NY). All statistical tests were made at a significant level of $p<0.05$. All error bars correspond to standard errors.

\section{Results}

\section{Incidence of SR response following LAS (Fig. 6)}

Analysis in the incidence of SR showed differential effects of PMAs vs. M1 stimulation depending on the LAS timing. A significant interaction between Stimulation $\times$ LAS condition was found $\left(F_{(1,36)}=7.246, p=0.011\right)$. Stratified analyses showed that SR incidence increased more after anodal M1 stimulation compared to cathodal PMAs stimulation when the LAS was at $-500 \mathrm{~ms}(p=0.001)$. In addition, after anodal M1 stimulation, SR incidence

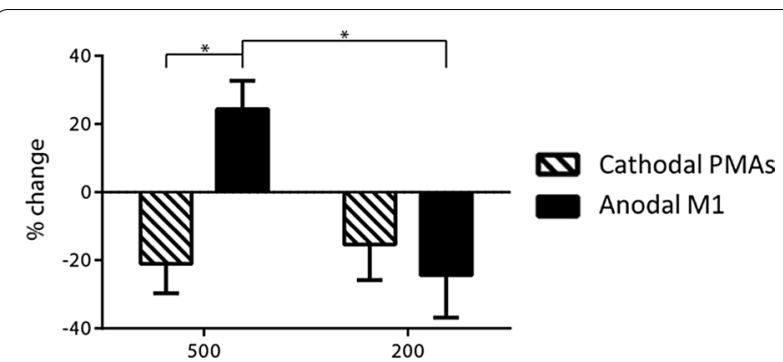

Fig. 6 Mean change ( \pm SE) of the incidence of SR response when the LAS was presented at 500 and $200 \mathrm{~ms}$ before the go cue

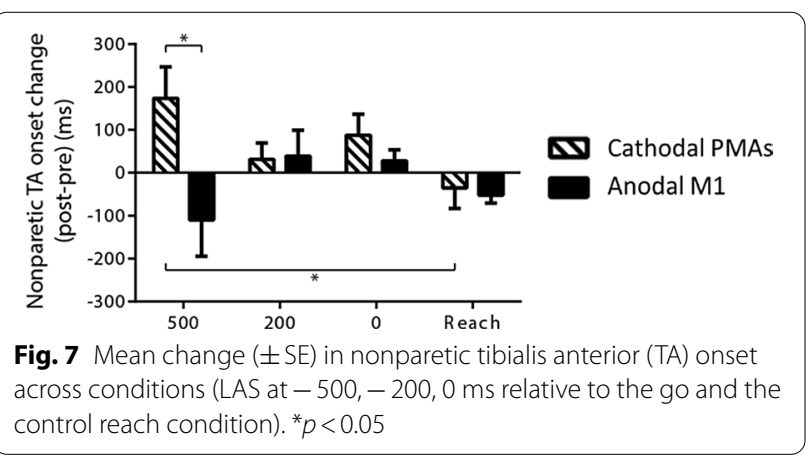

increased when the LAS was at $-500 \mathrm{~ms}$ but decreased when the LAS was at $-200 \mathrm{~ms}(p=0.004)$. In contrast, cathodal PMAs stimulation caused a decrease in the SR incidence at both LAS time points $-500 \mathrm{~ms}$ and $-200 \mathrm{~ms}$. This suggests a differential effect of cathodal PMAs vs. anodal M1 stimulation on SR incidence depending on the LAS timing.

\section{APA-reach performance}

There was a significant Stimulation $\times$ LAS condition interaction $\left(F_{(3,61.587)}=3.146, p=0.017\right)$ on nonparetic tibialis anterior onset. Stratified analyses showed that there was a significant effect of Stimulation site when the LAS was at $-500 \mathrm{~ms}(p=0.018)$. Specifically, the nonparetic tibialis anterior onset was later after cathodal PMAs stimulation than after anodal M1 stimulation at LAS time point $-500 \mathrm{~ms}$ (Fig. 7). In addition, after cathodal PMAs stimulation, there was a larger increase of nonparetic tibialis anterior onset time when the LAS was at $-500 \mathrm{~ms}$ compared to the control reach condition where there was no LAS applied $(p=0.045)$.

There was a significant Stimulation $\times$ LAS condition interaction $\left(F_{(3,72)}=4.708, p=0.005\right)$ in anterior deltoid onset (Fig. 8). Stratifies analyses showed that there was effect of LAS condition after anodal M1 stimulation showing that a decrease in anterior deltoid onset when the LAS was at $-500 \mathrm{~ms}$ compared to when the LAS was at $0 \mathrm{~ms}(p=0.026)$. Stratified analyses also showed 


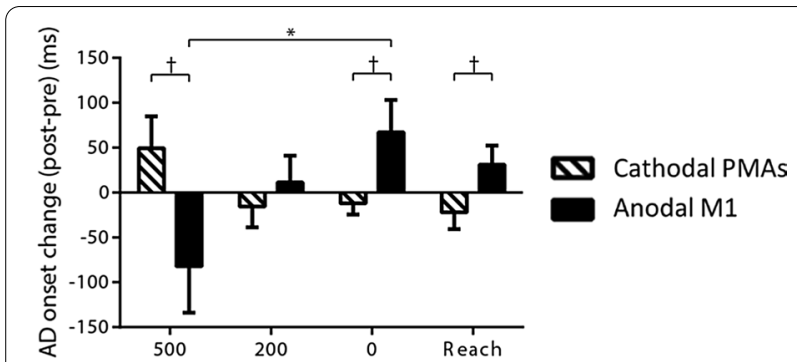

Fig. 8 Mean change $( \pm S E)$ in anterior deltoid (AD) onset across conditions (LAS at $-500,-200,0 \mathrm{~ms}$ relative to the go and the control reach condition). ${ }^{*} p<0.05$ and ${ }^{\dagger} p<0.1$

that there were effects of Stimulation when the LAS was at $-500 \mathrm{~ms}(p=0.05), 0 \mathrm{~ms}(p=0.053)$, and control reach condition $(p=0.058)$ although the difference was outside the significance cutoff. When the LAS was at $-500 \mathrm{~ms}$, the increase of anterior deltoid onset was larger after cathodal PMAs stimulation than anodal M1 stimulation. However, when the LAS was at $0 \mathrm{~ms}$ or in the control reach condition, the increase of anterior deltoid was larger after anodal M1 stimulation than cathodal PMAs stimulation. There were no main effects of Stimulation,
LAS condition, and Stimulation $\times$ LAS condition interaction on other APA-reach performance variables.

\section{Trunk contribution during movement execution}

A significant main effect of Stimulation was found on the trunk flexion $\left(F_{(1,66)}=8.622, p=0.005\right)$ and the trunkpelvic rotation difference $\left(F_{(1,75)}=4.721, p=0.033\right)$. The trunk flexion and the trunk-pelvic rotation difference had a larger increase after anodal M1 stimulation compared to after cathodal PMAs stimulation (Fig. 9a, b). Marginal main effects of Stimulation on trunk rotation was found (trunk rotation: $\left.F_{(1,66)}=3.294, p=0.074\right)$. After anodal M1 stimulation, there was a trend of greater increase in the trunk rotation compared to after cathodal PMAs stimulation (Fig. 9c). No significant main effects of LAS condition and Stimulation $\times$ LAS condition interaction were found for the above outcome variables.

\section{Neurophysiological measurement}

The number of subjects who we were able to retreive MEPs for biceps brachii was 6 and for tibialis anterior was 8 subjects for the anodal M1 stimulation. For the cathodal PMAs stimulation, we were able to retreive MEPs in 5 subjects for biceps brachii and in 7 subjects for a
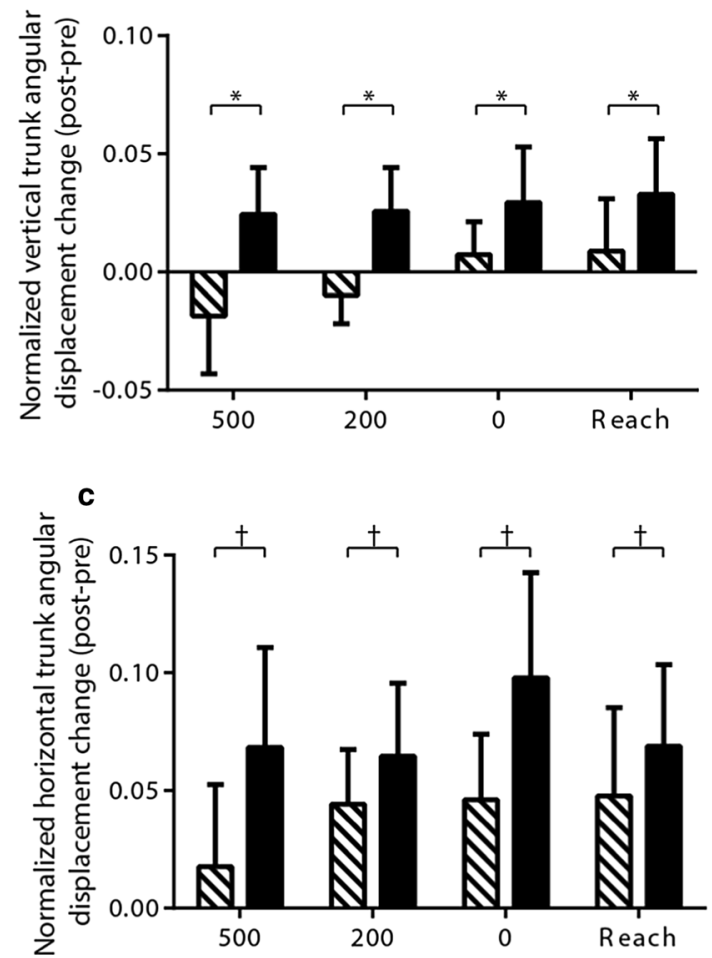

b

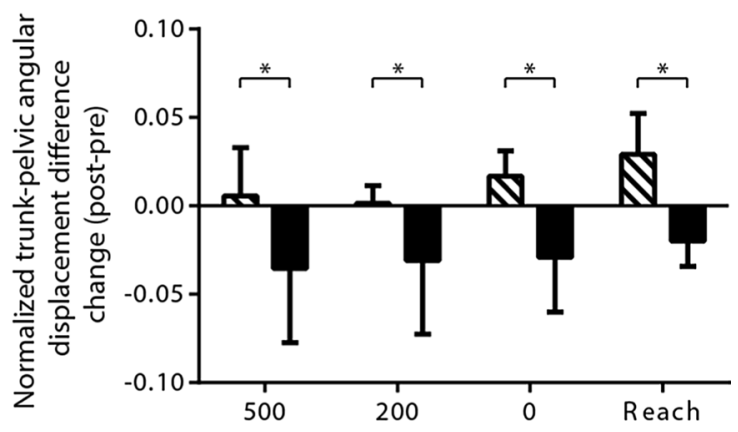

Q Cathodal PMAs

Anodal M1

Fig. 9 Mean change $( \pm S E)$ in a trunk flexion, b trunk-pelvic rotation difference, and c trunk rotation across conditions (LAS at - $500,-200,0$ ms relative to the go and the control reach condition). ${ }^{*} p<0.05$ and ${ }^{\dagger} p<0.1$ 
tibialis anterior. No significant difference in MEP amplitudes before and after tDCS was found.

\section{Discussion}

The findings that anodal tDCS over M1 was more effective in increasing the SR incidence than cathodal tDCS over PMAs in individuals with stroke was unexpected. It has been suggested that PMRF is critically involved in generating posture responses $[9,10]$ and in the SR responses [19].We proposed that the abnormal hyperexcitability in PMAs due to chronic stroke may lead to excessive inhibitory input via the cortico-reticulospinal pathway, and consequently impairs posture and movement planning, preparation, and execution. Therefore, down-regulating the hyperexcitability in PMAs by applying cathodal tDCS over these regions may restore the inhibitory input from PMAs to the PMRF (Fig. 10a). However, opposite to our hypothesis, our findings showed an increase in SR incidence and a decrease in muscle activation onset latency when the LAS was applied at $-500 \mathrm{~ms}$ after anodal M1 stimulation compared to cathodal PMAs stimulation in individuals with stroke (Fig. 10b). This is consistent in part with Nonnekes et al. [19] who showed a decrease in reaction time irrespective of whether or not an LAS was given at the imperative stimulus after anodal M1 stimulation. The author concluded that the subcortical structures can possibly be facilitated by an enhancement of the corticoreticular drive or by direct excitations caused by the applied current. One animal study also showed that tDCS over the motor cortex of anesthetized cats facilitated subcortical structures either directly or indirectly [33]. Moreover, Wagner and colleagues showed possible direct subcortical facilitation from the spread of current during tDCS application and importantly the current density distributions were different in the stroke model [34]. It is possible that application of anodal tDCS over M1 facilitates directly or indirectly the subcortical structures such as PMRF. With our stimulation paradigm, rather than remediating the excessive inhibitory input from the PMAs onto the subcortical brainstem by applying cathodal tDCS over PMAs, the direct or indirect subcortical facilitation from anodal tDCS over the region of M1 may be more effective modulating brainstem neuronal excitability and in turn improving movement planning and preparation. Another plausible explanation is that given that PMAs include a larger cortical area compared to M1, a higher intensity or longer duration of tDCS is required to induce changes in PMAs. Although the findings from this study are not consistent with our hypothesis, our conceptual model does not mutually exclude the possible subcortical facilitation mechanisms.
Another factor that influenced the effect of tDCS on the incidence of SR response was the LAS timing. A greater increase in the incidence of SR responses following anodal M1 stimulation was only found at the LAS time point $-500 \mathrm{~ms}$ but not at $-200 \mathrm{~ms}$. One possible explanation is that during the time course of movement planning and preparation, the excitability at the cortical and subcortical levels changes gradually. During preparation for movement there are two pathways controlling spinal motorneuronal circuitry in order to preplan the movement and avoid premature release of the movement [7]. The excitatory input which originates from PMAs and relays via $\mathrm{M} 1$ to spinal cord transmits task-related information while the global inhibition originates from PMAs and relays via subcortical level to spinal circuity (i.e., cortico-reticulospinal pathway) prevents premature release of a motor action. It has been suggested that the corticospinal excitability during movement planning and preparation undergoes progressive changes due to global inhibition [35]. The dynamic changes of inhibitory inputs onto spinal motorneuronal circuitry during movement planning and preparation may lead to differential effects of tDCS on the incidence of SR responses depending on the LAS timing.

There were no detectable neurophysiological change of MEP amplitudes following PMAs and M1 stimulation but posture and movement planning, preparation, and execution were modified after tDCS. One possible reason for non-significant changes in MEP amplitudes is inter-individuals response variability. Previous studies have reported that the effects of tDCS on MEP amplitudes elicited by single-pulse TMS as a measure for corticospinal excitability are highly variable $[36,37]$. Another possible reason is that the changes in cortical excitability after tDCS are the result of intracortical facilitation or inhibition not corticospinal excitability as shown in MEP amplitudes elicited by single-pulse TMS. One study by Nitsche et al. [38] used paired-pulse TMS with different interstimulus interval and found that following tDCS intracortical inhibition and facilitation were modified. This may suggest that single-pulsed MEP amplitude may not be a preferred indicator of neurophysiological changes obtained by tDCS.

Our findings show a differential effect of cathodal PMAs vs anodal M1 stimulation on trunk contribution during reaching execution across LAS conditions. Generally, we found that there was a greater increase in trunk movement after anodal M1 compared to cathodal PMAs stimulation. One possible explanation is that the anodal electrode placement over M1 stimulation included the trunk representation since it was at the midpoint of hotspots of biceps brachii and tibialis anterior. Based on the homunculus map of a human brain, 


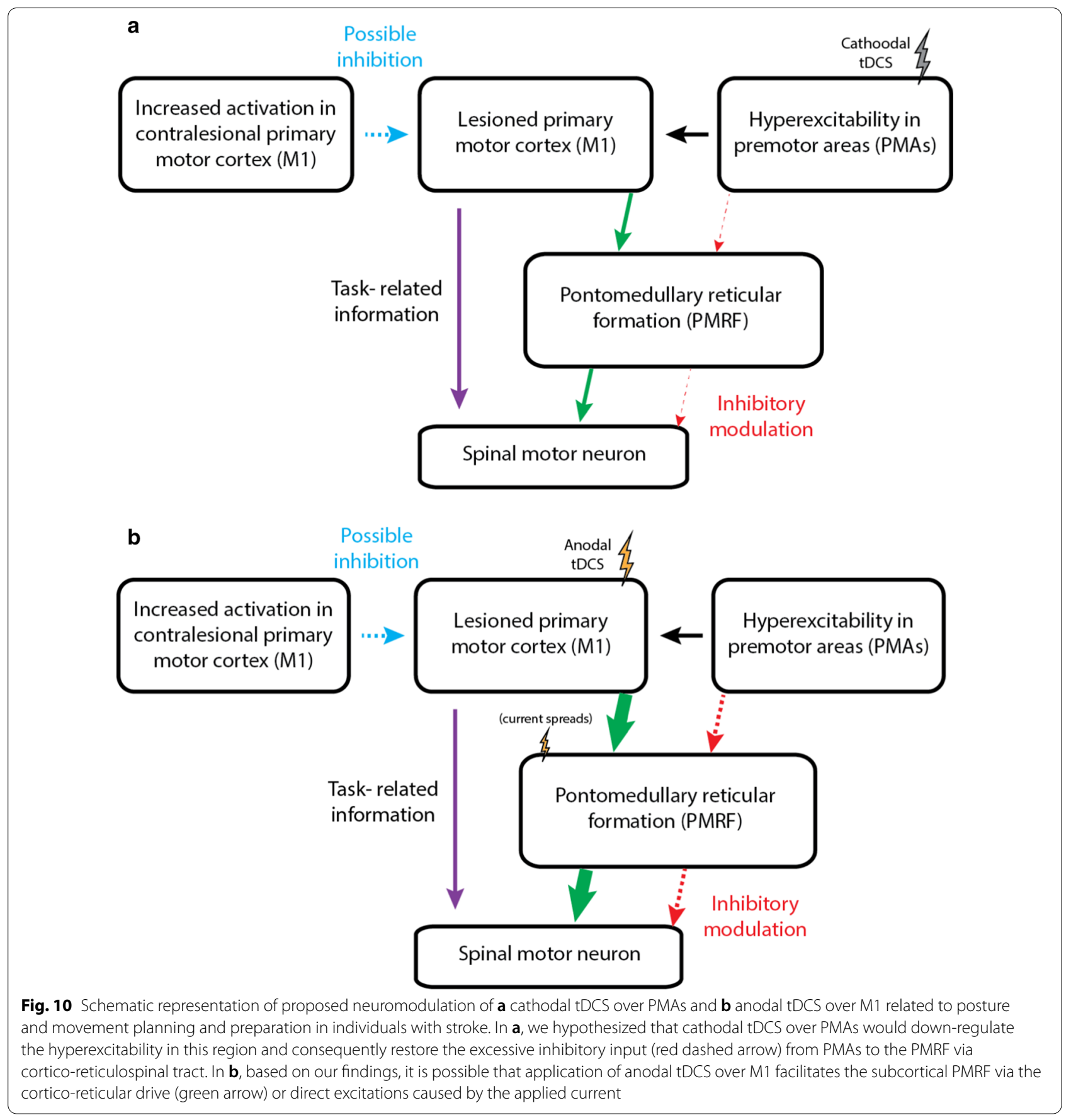

the area representing the trunk is in the middle of arm and leg areas. Thus, the anodal M1 stimulation may also affect trunk performance. Another plausible explanation is the subcortical facilitation described in the previous section. Since the PMRF is known to be involved in generating compensatory postural responses $[9,10]$, the direct or indirect facilitation induced by anodal M1 stimulation may increase the excitability in the
PMRF and subsequently alter trunk involvement during reaching.

One major challenge of our study was that we aimed to modulate PMAs and M1 separately. Even though small tDCS electrodes $\left(15 \mathrm{~cm}^{2}\right)$ were used over both target areas in an attempt to increase the focality of stimulation, the possibility that during PMAs stimulation, M1 region was also partly stimulated can not be ruled out, and vice 
versa. Nevertheless, differential effects of cathodal PMAs vs. anodal M1 stimulation on variables of posture, movement planning, preparation and execution were demonstrated in the present study and provide credible evidence that modulation of these two areas by tDCS is plausible. Another limitation is that the absence of detectable MEP amplitudes changes following tDCS. The difficulty to record MEPs data in $20-30 \%$ of our stroke subjects, inter-individuals response variability, and inability to capture changes in cortical excitability by single-pulsed TMS protocol possibly contributed to nonsignificant changes in MEP amplitudes. Future studies should also consider measuring cortical excitability changes following tDCS with a more comprehensive assessment by TMS such as cortical silent period in order to detect the modulation effect of tDCS.

\section{Conclusions}

The present results show that following the application of cathodal and anodal tDCS over the region of the PMAs or M1, respectively, "stimulation"-specific changes were observed in posture and movement planning, preparation and execution in individuals with stroke. We also provide novel evidence that stroke-related deficits in movement planning and preparation as shown by an abnormal absence of SR responses can be improved by application of anodal tDCS over lesioned M1 and the enhancement effects are depending on the timing of the LAS. It is possible that either direct or indirect subcortical facilitation resulting from the anodal tDCS over M1 may offer a new neuromodulatory target to remediate the imbalance in neuronal excitability between PMAs and subcortical brainstem level, which in turn improve the posture and movement planning, preparation, and execution in individuals with stroke.

\begin{abstract}
Abbreviations
BB: Biceps brachii; TA: Tibialis anterior; MVIC: Maximum voluntary isometric contraction; AMT: Active motor threshold; SMA: Supplementary motor area; PMC: Premotor cortex; APA: Anticipatory postural adjustment; LAS: Loud acoustic stimulus; M1: Primary motor area; MEP: Motor evoked potentials; PMAs: Premotor areas; PMRF: Pontomedullary reticular formation; SR: StartReact; tDCS: Transcranial direct current stimulation; TMS: Transcranial magnetic stimulation.
\end{abstract}

\section{Authors' contributions}

CY conceived and designed research; CY and SMW performed experiments; CY analyzed data; RAC developed codes for data analysis; LM provided consultation about statistical analysis; $C Y$ interpreted results of experiments; $C Y$ prepared figures; CY drafted manuscript; CY, MWR, AG, LM, and SMW edited and revised manuscript; All authors read and approved the final manuscript.

\section{Funding}

This study was supported by the American Heart Association 16PRE29970004 to $\mathrm{CY}$ and the University of Maryland Claude D. Pepper-OAIC NIH/NIA Grant P30AG028747 to MWR.

\section{Availability of data and materials}

The datasets used and/or analysed during the current study are available from the corresponding author on reasonable request.

\section{Ethics approval and consent to participate}

The study was approved by the Institutional Review Board at the University of Maryland Baltimore (HP-00064894). Written informed consent was obtained from all individual participants included in the study.

\section{Consent for publication}

Not applicable.

\section{Competing interests}

The authors declare that they have no competing interests.

\section{Author details}

${ }^{1}$ Department of Physical Therapy and Rehabilitation Science, University of Maryland School of Medicine, 100 Penn Street, Baltimore, MD 21201, USA.

${ }^{2}$ Department of Epidemiology and Public Health, University of Maryland School of Medicine, Baltimore, MD 21201, USA. ${ }^{3}$ Present Address: Rehabilitation Research Program, Vancouver Coastal Health Research Institute, 4255 Laurel Street, Vancouver, BC V5Z2G9, Canada. ${ }^{4}$ Department of Physical Therapy, University of British Columbia, Vancouver, BC V6T1Z3, Canada. ${ }^{5}$ Lewis Human Performance Lab, Department of Exercise Science, Lebanon Valley College, Annville, PA 17003, USA. ${ }^{6}$ Division of Health, Business, Technology and Science, Frederick Community College, 7932 Oppossumtown Pike, Frederick, MD 21702, USA.

Received: 23 June 2020 Accepted: 9 December 2020

Published online: 07 January 2021

\section{References}

1. Carlsen AN, Maslovat D, Lam MY, Chua R, Franks IM. Considerations for the use of a startling acoustic stimulus in studies of motor preparation in humans. Neurosci Biobehav Rev. 2011;35(3):366-76.

2. Carlsen AN, Maslovat D, Franks IM. Preparation for voluntary movement in healthy and clinical populations: evidence from startle. Clin Neurophysiol. 2012;123(1):21-33.

3. McCombe Waller S, Yang CL, Magder L, Yungher D, Gray V, Rogers MW. Impaired motor preparation and execution during standing reach in people with chronic stroke. Neurosci Lett. 2016;630:38-44.

4. Yang $\mathrm{CL}$, Creath RA, Magder L, Rogers MW, McCombe WS. Impaired posture, movement preparation, and execution during both paretic and nonparetic reaching following stroke. J Neurophysiol. 2019;121(4):1465-77.

5. Viallet F, Massion J, Massarino R, Khalil R. Coordination between posture and movement in a bimanual load lifting task: putative role of a medial frontal region including the supplementary motor area. Exp Brain Res. 1992:88(3):674-84.

6. Chang WH, Tang PF, Wang YH, Lin KH, Chiu MJ, Chen SH. Role of the premotor cortex in leg selection and anticipatory postural adjustments associated with a rapid stepping task in patients with stroke. Gait Posture. 2010;32(4):487-93.

7. Cohen O, Sherman E, Zinger N, Perlmutter S, Prut Y. Getting ready to move: transmitted information in the corticospinal pathway during preparation for movement. Curr Opin Neurobiol. 2010;20(6):696-703.

8. Flöel A. tDCS-enhanced motor and cognitive function in neurological diseases. Neurolmage. 2014;85(Pt 3):934-47.

9. Schepens B, Drew T. Strategies for the integration of posture and movement during reaching in the cat. J Neurophysiol. 2003;90(5):3066-86.

10. Yakovenko S, Drew T. A motor cortical contribution to the anticipatory postural adjustments that precede reaching in the cat. J Neurophysiol. 2009;102(2):853-74

11. Ward NS, Brown MM, Thompson AJ, Frackowiak RS. Neural correlates of outcome after stroke: A cross-sectional fMRI study. Brain. 2003;126(Pt 6):1430-48.

12. Nitsche MA, Fricke K, Henschke U, Schlitterlau A, Liebetanz D, Lang $\mathrm{N}$, et al. Pharmacological modulation of cortical excitability shifts 
induced by transcranial direct current stimulation in humans. J Physiol. 2003;553(Pt 1):293-301.

13. Fregni F, Boggio PS, Mansur CG, Wagner T, Ferreira MJ, Lima MC, et al. Transcranial direct current stimulation of the unaffected hemisphere in stroke patients. NeuroReport. 2005;16(14):1551-5.

14. Hummel F, Celnik P, Giraux P, Floel A, Wu WH, Gerloff C, et al. Effects of non-invasive cortical stimulation on skilled motor function in chronic stroke. Brain. 2005;128(Pt 3):490-9.

15. Madhavan S, Weber KA 2nd, Stinear JW. Non-invasive brain stimulation enhances fine motor control of the hemiparetic ankle: Implications for rehabilitation. Exp Brain Res. 2011;209(1):9-17.

16. Tanaka S, Takeda K, Otaka Y, Kita K, Osu R, Honda M, et al. Single session of transcranial direct current stimulation transiently increases knee extensor force in patients with hemiparetic stroke. Neurorehabil Neural Repair. 2011;25(6):565-9.

17. Sohn MK, Jee SJ, Kim YW. Effect of transcranial direct current stimulation on postural stability and lower extremity strength in hemiplegic stroke patients. Ann Rehabil Med. 2013;37(6):759-65.

18. Stagg CJ, Bachtiar V, O'Shea J, Allman C, Bosnell RA, Kischka U, et al. Cortical activation changes underlying stimulation-induced behavioural gains in chronic stroke. Brain. 2012;135(Pt 1):276-84.

19. Nonnekes J, Arrogi A, Munneke MA, van Asseldonk EH, Oude Nijhuis LB, Geurts AC, et al. Subcortical structures in humans can be facilitated by transcranial direct current stimulation. PLoS ONE. 2014;9(9):e107731.

20. Gladstone DJ, Danells CJ, Black SE. The Fugl-Meyer assessment of motor recovery after stroke: A critical review of its measurement properties. Neurorehabil Neural Repair. 2002;16(3):232-40.

21. Hayduk-Costa G, Drummond NM, Carlsen AN. Anodal tDCS over SMA decreases the probability of withholding an anticipated action. Behav Brain Res. 2013;257:208-14.

22. Picard N, Strick PL. Imaging the premotor areas. Curr Opin Neurobiol. 2011;11(6):663-72.

23. Melgari JM, Pasqualetti P, Pauri F, Rossini PM. Muscles in "concert": study of primary motor cortex upper limb functional topography. PLOS ONE. 2008;3(8):e3069.

24. Khaslavskaia S, Ladouceur M, Sinkjaer T. Increase in tibialis anterior motor cortex excitability following repetitive electrical stimulation of the common peroneal nerve. Exp Brain Res. 2002;145(3):309-15.

25. Rothwell JC, Hallett M, Berardelli A, Eisen A, Rossini P, Paulus W. Magnetic stimulation: motor evoked potentials: the international federation of clinical neurophysiology. Clin Neurophysiol Suppl. 1999;52:97-103.

26. Mackinnon CD, Bissig D, Chiusano J, Miller E, Rudnick L, Jager C, et al. Preparation of anticipatory postural adjustments prior to stepping. J Neurophysiol. 2007;97(6):4368-79.
27. Siegmund GP, Inglis JT, Sanderson DJ. Startle response of human neck muscles sculpted by readiness to perform ballistic head movements. J Physiol. 2001;535(Pt 1):289-300.

28. Carlsen AN, Chua R, Inglis JT, Sanderson DJ, Franks IM. Startle response is dishabituated during a reaction time task. Exp Brain Res. 2003;152(4):510-8.

29. Roy SH, De Luca G, Cheng MS, Johansson A, Gilmore LD, De Luca CJ. Electro-mechanical stability of surface EMG sensors. Med Biol Eng Comput. 2007:45(5):447-57.

30. Eames MHA, Cosgrove A, Baker R. Comparing methods of estimating the total body centre of mass in three-dimensions in normal and pathological gaits. Hum Mov Sci. 1999;18:637-46.

31. SENIAM. Surface ElectrodeMyoGraphy for the non-invasive assessment of muscles. http://www.seniam.org/. Accessed 23 Jan 2020.

32. Hodges PW, Bui BH. A comparison of computer-based methods for the determination of onset of muscle contraction using electromyography. Electromyogr Clin Neurophysiol. 1996;101(6):511-9.

33. Bolzoni F, Pettersson LG, Jankowska E. Evidence for long-lasting subcortical facilitation by transcranial direct current stimulation in the cat. J Physiol. 2013;591(13):3381-99.

34. Wagner T, Fregni F, Fecteau S, Grodzinsky A, Zahn M, Pascual-Leone A. Transcranial direct current stimulation: a computer-based human model study. Neurolmage. 2007;35(3):1113-24.

35. van Elswijk G, Schot WD, Stegeman DF, Overeem S. Changes in corticospinal excitability and the direction of evoked movements during motor preparation: a TMS study. BMC Neurosci. 2008;9:51.

36. Wiethoff S, Hamada M, Rothwell JC. Variability in response to transcranial direct current stimulation of the motor cortex. Brain Stimul. 2014;7(3):468-75.

37. Lopez-Alonso V, Fernandez-Del-Olmo M, Costantini A, GonzalezHenriquez JJ, Cheeran B. Intra-individual variability in the response to anodal transcranial direct current stimulation. Clin Neurophysiol. 2015;126(12):2342-7.

38. Nitsche MA, Seeber A, Frommann K, Klein CC, Rochford C, Nitsche MS, et al. Modulating parameters of excitability during and after transcranial direct current stimulation of the human motor cortex. J Physiol. 2005;568(Pt 1):291-303.

\section{Publisher's Note}

Springer Nature remains neutral with regard to jurisdictional claims in published maps and institutional affiliations.
Ready to submit your research? Choose BMC and benefit from:

- fast, convenient online submission

- thorough peer review by experienced researchers in your field

- rapid publication on acceptance

- support for research data, including large and complex data types

- gold Open Access which fosters wider collaboration and increased citations

- maximum visibility for your research: over $100 \mathrm{M}$ website views per year

At BMC, research is always in progress.

Learn more biomedcentral.com/submissions 